AperTO - Archivio Istituzionale Open Access dell'Università di Torino

A review of the recent advances in neuroimaging of frontotemporal lobar degeneration

This is a pre print version of the following article:

Original Citation:

Availability:

This version is available http://hdl.handle.net/2318/1619728

since 2016-12-01T21:14:14Z

Published version:

DOI:10.23736/S0390-5616.16.03281-1

Terms of use:

Open Access

Anyone can freely access the full text of works made available as "Open Access". Works made available under a Creative Commons license can be used according to the terms and conditions of said license. Use of all other works requires consent of the right holder (author or publisher) if not exempted from copyright protection by the applicable law. 


\section{A review of recent advances in neuroimaging of Frontotemporal Lobar Degeneration}

D’Agata $\mathrm{F}^{\mathrm{a} \bowtie}$, Orsi $\mathrm{L}^{\mathrm{b}}$, Cicerale $\mathrm{A}^{\mathrm{a}}$, Rubino $\mathrm{E}^{\mathrm{c}}$, Rainero I ${ }^{\mathrm{bc}}$, Bergui $\mathrm{M}^{\mathrm{ad}}$, Pinessi $\mathrm{L}^{\mathrm{bc}}$

a LabNI, Department of Neuroscience, University of Turin, Italy

b Department of Neuroscience and Mental Health, AOU Città della Salute e della Scienza, Turin, Italy

c Department of Neuroscience, Neurology Section, University of Turin, Turin, Italy

d Department of Neuroscience, Neuroradiology Section, AOU Città della Salute e della Scienza, Turin, Italy

${ }^{凶}$ corresponding author

Federico D’Agata, Ph.D.

Department of Neuroscience, Via Cherasco, 15 - 10126, Torino

Tel: +390116636298 ; Fax: +390116963487

e-mail: federico.dagata@unito.it

Keywords: Frontotemporal Lobar Degeneration; Diagnostic imaging; Neuroimaging; Tomography, Emission-Computed; Magnetic Resonance Imaging

Abstract: 200 words; main text: 6000 words; figures: 2 b/w; tables: 3 


\section{Abstract}

Backgrounds: The terms frontotemporal lobar degeneration (FTLD) indicate a large set of neurodegenerative diseases, heterogeneous in their genetic, pathologic and clinical aspects.

Objectives: This review will focus on the most recent contribution of neuroimaging tools on the diagnosis, characterization and pathogenesis of FTLD.

Data sources: PubMed, Scopus, Ovid.

Study eligibility criteria: recent papers published in English in the last 3 years.

Results: We found 91 papers of interest and reviewed their contents, finding in particular 4 major topics: the contribution of neuroimaging on the differential diagnosis; patients' functional characterization; new neuroimaging tools under development and presymptomatic genetic forms.

Conclusions: Neuroimaging techniques have shown to be useful supporting tools in diagnosis, even if not always determinant to reach a conclusive decision, and quite important to identify phenocopies. At the moment there is not a neuroimaging biomarker that could track the progressive course of dementias and the effect of therapies, but it is possible that in the future Diffusion Tensor Imaging and molecular imaging could fill this void. Monitoring in vivo the evolution of the pathology for at least 5 years is essential, which would only be possible in a large multicentric study, while asymptomatic forms would require even longer observation periods. 


\section{Introduction}

The terms frontotemporal lobar degeneration (FTLD) indicate a large set of neurodegenerative diseases, very heterogeneous in their genetic, pathologic and clinical aspects ${ }^{1}$ that selectively targets the frontal and temporal brain lobes, which become progressively hypoperfused and atrophic. In the last 3 years the scientific community has shown an exponentially increasing interest in describing and characterizing FTLD. It is however still unclear whether the different genetic, clinical and pathological manifestation of FTLD are different expressions of a single underlying condition or if they are different pathologies that damage the same groups of cerebral areas or functional networks.

While FTLD is rarer than AD, it is the second cause of presenile dementia $(<65$ years $)$ and therefore of great interest, considering the social and economic costs of the loss of functionality. In $60 \%$ of FTLD patients the onset is between 45 and 64 years, in $10 \%$ before 45 years and in $30 \%$ after 65 th year of life ${ }^{2}$. Overall, FTLD is the third most common cause of dementia after AD and Dementia with Lewy bodies (DLB) and constitutes 5-10\% of all dementias confirmed by histopathological examination. The incidence of FTLD is estimated to be 3 to 4 per 100000 personyear, its prevalence in the 45-64 years population is $15-22$ per 100000 in the USA, 15 per 100000 in the UK and 22 per 100000 in Italy ${ }^{2}$. The progression of the disease is on average 7-11 years, and the diagnosis usually is formulated 3-4 years after the onset. The average survival from the onset (911 years) is extremly variable between different forms, with some patients that can live more than 20 years $^{2-4}$. The main causes of death are cardiopulmonary collapse and cachexia.

The main clinical variants of FTLD ${ }^{1}$ are the behavioral (bvFTD, behavior variant FTD) and the linguistic ones (Primary Progressive Aphasia, PPA). PPA can be categorized in 3 subtypes ${ }^{5,6}$ : the non-fluent/agrammatic variant, also called progressive non-fluent aphasia (nfPPA or PNFA), semantic dementia or semantic variant (SD o svPPA), logophenic variant or logophenic progressive 
aphasia (lvPPA o LPA). Table I report the main clinical characteristics of the different variants of FTLD (lvPPA was not reported as generally is associated with AD).

Table I. Clinical features of FTLD

\begin{tabular}{|c|c|c|c|c|}
\hline Syndrome & Behaviour & Cognition & Functional ability & $\begin{array}{c}\text { Motor } \\
\text { symptoms }\end{array}$ \\
\hline bvFTD & $\begin{array}{l}\text { Disinhibition; apathy; } \\
\text { loss of empathy; } \\
\text { stereotyped behaviours; } \\
\text { hyperorality }\end{array}$ & $\begin{array}{l}\text { Executive deficits; } \\
\text { impaired social } \\
\text { cognition; } \\
\text { lack of insight }\end{array}$ & $\begin{array}{l}\text { Activities of daily living } \\
\text { impaired by executive } \\
\text { dysfunction, impaired } \\
\text { persistence and impulse } \\
\text { control }\end{array}$ & $\begin{array}{l}\text { Signs of PSPS, } \\
\text { CBS or MND }\end{array}$ \\
\hline nfPPA & $\begin{array}{l}\text { Relative intact until } \\
\text { late }\end{array}$ & $\begin{array}{l}\text { Non fluent language } \\
\text { production, some } \\
\text { anomia }\end{array}$ & $\begin{array}{l}\text { Activities of daily living } \\
\text { impaired by deficits of verbal } \\
\text { communication }\end{array}$ & $\begin{array}{l}\text { Signs of PSPS, } \\
\text { CBS or MND; } \\
\text { apraxia of speech }\end{array}$ \\
\hline svPPA & $\begin{array}{l}\text { Loss of empathy; } \\
\text { mental rigidity; } \\
\text { ritualistic behavior; } \\
\text { dietary change }\end{array}$ & $\begin{array}{l}\text { Impaired linguistic } \\
\text { comprehension, some } \\
\text { anomia }\end{array}$ & $\begin{array}{l}\text { Activities of daily living } \\
\text { impaired by semantic deficits } \\
\text { and problems with planning }\end{array}$ & Intact until late \\
\hline
\end{tabular}

From a neuropathological point of view, FTLD can be classified by the accumulated protein (tau, TDP-43, FUS, other) and divided into subtypes by considering the characteristics of the protein agglomerates ${ }^{8}$. In particular, proteins described in the previous sections are involved in $90-95 \%$ of cases: FTLD-tau accounts for $40 \%$ of cases, FTLD-TDP accounts for $40-50 \%$ of cases and the FTLD-FUS accounts for 5-10\% of the remaining cases.

In about $40 \%$ of cases there is familiarity for FTLD, but only in $10-30 \%$ of the cases it is possible to identify an autosomal dominant transmission of the illness. To the present day several genes whose mutation is linked to FTLD have been identified, and the main three are: i) chromosome 9 open reading frame $72(C 9 O R F 72)$, ii) microtubule-associated protein tau (MAPT) and iii) progranulin $(G R N)$. Taken together, these three mutations explain about $80 \%$ of autosomal dominant familial cases $^{9}$. 
Diagnosis of FTLD is often complicated, and generally the diagnostic criteria for FTLD are articulated on three levels (see ${ }^{10}$ for bvFTD validated consensus diagnostic criteria and ${ }^{11}$ for some useful indications about PPA): for a possible diagnosis there must be behavioral and/or neuropsychological changes, for a probable diagnosis there is the additional requirement of cortical alteration demonstrated by neuroimaging (Computed Tomography, CT; Magnetic Resonance Imaging, MRI; Positron Emission Tomography, PET; Single Photon Emission Computerized Tomography, SPECT). To reach the certainty of the diagnosis, there must be either neuropathological or genetic findings suggestive of FTLD.

Even when strictly abiding by the diagnostic criteria, clinical diagnosis of possible FTLD contain up to $10-20 \%$ of undiagnosed $\mathrm{AD}$ cases, as $\mathrm{AD}$ can have atypical onsets ${ }^{12}$. Different variant of FTLD can coexist in their clinical progression and comorbidity with motor symptoms and neurodegenerative disease as Motor Neuron Disease (MND), in particular Amyotrophic Lateral Sclerosis (ALS), or atypical parkinsonisms ${ }^{13}$ as Corticobasal Syndrome (CBS), or Progressive Supranuclear Palsy Syndrome (PSPS).

Considering all these diagnostic challenges, every serious tentative of diagnosis should be supported by a neuroimaging demonstration of frontal or temporal involvement.

Another reason to consider the clinical description not sufficient to identify FTLD is the existence of phenocopies ${ }^{14}$ : that are patients (generally males) with symptoms that closely mimic bvFTD, but with a positive prognosis and relatively stable clinical pictures. The majority of studies, focused on discriminating different clinical subgroups or groups with different dementias (FTLD, DLB and AD) with neuroimaging, achieved good or excellent results ${ }^{15}$. A focal brain involvement of the frontal and temporal lobes, typically hypoperfusion or atrophy, but also other kind of alterations (e.g. hypometabolism, white matter damage, etc...), is an important supportive element to a correct diagnosis.

Currently there is not a clear understanding of the etiopathogenesis of this disease, nor medications or treatments able to cure, but some drugs that could act on pathogenic mechanisms are under 
investigation. While waiting for more effective therapeutic tools, the main goal of the scientific community is to better understand the pathology and to obtain useful markers for early diagnosis ${ }^{9}$. This review of the literature was systematic, but qualitative, and focused on the last 3 years publications about FTLD and clinical neuroimaging, a topic chosen for its relevance and newsworthy.

\section{Methods}

We followed the PRISMA guidelines recommendations for the search, the selection and the reporting of the relevant papers. We chose to use the following scientific references resources: Scopus, Ovid, PubMed and MEDLINE. We searched for articles published from January 2012 up to December 2014 (time limits), using combinations of the following keywords MeSH: Frontotemporal Lobar Degeneration; Diagnostic imaging; Neuroimaging; Image Interpretation, Computer-Assisted; Tomography, Emission-Computed; Tomography; Magnetic Resonance Imaging. Searches were limited to articles published in English. Frontotemporal Lobar Degeneration was always in the search queries in combination with logic AND with at least one other keyword. All the queries were pooled together, removing the duplicates. The resulting references were screened to keep only the papers concerning clinical neuroimaging. Further references were retrieved manually by reviewing the bibliographies of the relevant publications.

\section{Results}

We found 528 papers through database searching and 12 more by looking at the references of the final selection. The number of records was 243 after removing duplicate entries and 146 records were excluded after looking as the titles and/or abstracts were not focused on clinical neuroimaging and FTLD (Fig. 1). 


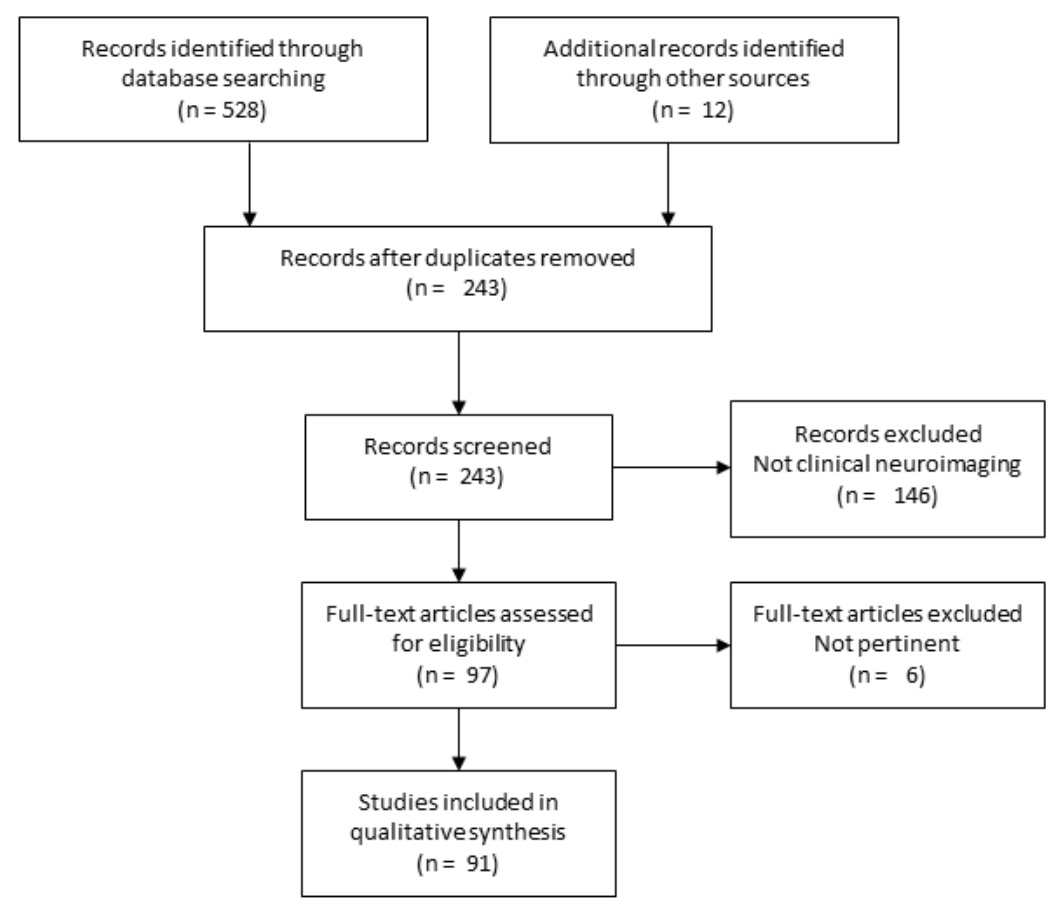

Figure 1. Flow diagram of studies selection for the current review.

The selected literature was classified in 4 groups:

1) The contribution of neuroimaging on the differential diagnosis of FTLD;

2) FTLD patients characterization with neuroimaging;

3) New neuroimaging tools under development for FTLD study;

4) The importance of the study of FTLD presymptomatic genetic forms.

Ninety-seven full-text articles were assessed for eligibility and 6 were discarded as not pertinent or not fitting in the 4 topics classification (Fig. 1).

Table II reports the level of evidence of the all 91 selected articles, according to the Oxford Centre for Evidence-based Medicine classification (March 2009, http://www.cebm.net/oxford-centreevidence-based-medicine-levels-evidence-march-2009). 
Table II. Level of evidence of articles

\begin{tabular}{|ll|}
\hline LOE & Reference number(s) \\
\hline $\mathbf{2 a}$ & $8,10,28,30,74,79$ \\
\hline $\mathbf{2 b}$ & $3,4,11,12,19,20,24,25,26,29,31,33,34,38,40,41,44,49,51,55,71,80,84,86$ \\
\hline $\mathbf{3 a}$ & $1,6,15,16,56,59,61$ \\
\hline $\mathbf{3 b}$ & $17,18,21,22,23,27,45,46,47,50,52,53,54,58,62,65,66,67,70,72,73,76,81,82,87$ \\
\hline $\mathbf{4}$ & $32,35,36,37,39,42,43,48,57,60,63,68,69,78,83,88,89,90,91$ \\
\hline $\mathbf{5}$ & $2,5,7,9,13,14,64,75,77,85$ \\
\hline
\end{tabular}

LOE = Level of Evidence from Oxford Centre for Evidence-based Medicine (March 2009)

http://www.cebm.net/oxford-centre-evidence-based-medicine-levels-evidence-march-2009

The reviewed studies were classified as $2 \mathrm{a}(7 \%), 2 \mathrm{~b}(26 \%), 3 \mathrm{a}(8 \%), 3 \mathrm{~b}(27 \%), 4(21 \%)$ and $5(11 \%)$.

\section{Discussion}

\section{Differential diagnosis}

The atrophy pattern in FTLD is usually more anterior and asymmetric than in AD. Table III reports the areas more interested by the different clinical, genetic and pathological forms ${ }^{16}$. While these patterns can be common, it is important to keep in mind that the variability within a single category (clinical, genetic or pathological) can be quite significant, as shown by a study ${ }^{17}$ that analyzed 66 subjects clinically diagnosed with bvFTD found 4 different atrophy patterns: frontal (21 subjects), temporal (6 subjects), fronto-temporal (12 subjects) and fronto-temporo-parietal one (27 subjects).

It should constantly be remembered that there can be partial overlap between pathologies and MRI alone could not be enough to make a diagnosis. For instance, a paper analyzing cortical thickness ${ }^{18}$ of $36 \mathrm{AD}$ and 24 FTLD, did not find any significant differences between the groups. Furthermore, studying the shape and the volume of hippocampus or temporal areas is extremely predictive of a neurodegenerative disease, but is not enough, when considered on its own, to discriminate between 
AD and FTLD ${ }^{19,20}$. While a thorough study of hippocampus and his sections can discriminate between different clinical forms, global indexes that can summarize the degree of anterior and posterior damage and of asymmetry can be more useful in clinical practice ${ }^{21}$.

Table III. Atrophy patterns in clinical, genetic, pathological variants of FTLD

\begin{tabular}{|c|c|c|c|c|c|c|c|c|c|}
\hline & PFC & PMC & $\mathrm{TL}$ med & TL lat & PL & $\mathrm{OL}$ & STR & CRB & LR \\
\hline \multicolumn{10}{|l|}{ Clinical } \\
\hline bvFTD & +++ & + & ++ & ++ & + & - & + & - & - \\
\hline nfPPA & - & ++ & - & - & - & - & + & - & $\mathrm{y}$ \\
\hline svPPA & + & - & +++ & +++ & - & - & + & - & - \\
\hline \multicolumn{10}{|l|}{ Genes } \\
\hline$M A P T$ & ++ & - & +++ & +++ & + & - & + & - & - \\
\hline$G R N$ & ++ & - & + & ++ & ++ & - & - & + & $\mathrm{y}$ \\
\hline C9orf72 & +++ & ++ & + & ++ & ++ & + & + & ++ & - \\
\hline \multicolumn{10}{|l|}{$\mathrm{Tau}$} \\
\hline PiD & +++ & + & ++ & ++ & + & - & + & - & $\mathrm{y}$ \\
\hline PSP & - & + & - & - & - & - & + & + & - \\
\hline CBD & + & ++ & - & - & + & - & ++ & - & - \\
\hline \multicolumn{10}{|l|}{ TDP } \\
\hline TDP A & +++ & - & + & ++ & ++ & - & - & + & $\mathrm{y}$ \\
\hline TDP B & ++ & + & + & + & - & - & + & - & - \\
\hline TDP C & + & - & +++ & +++ & - & - & + & - & $\mathrm{y}$ \\
\hline FUS & ++ & - & + & + & - & - & +++ & - & - \\
\hline
\end{tabular}

Adapted from ${ }^{16}$, bvFTD $=$ behavioral variant frontotemporal dementia, nfPPA $=$ non fluent variant primary progressive aphasia, svPPA = sementic variant primary progressive aphasia, $\mathrm{PiD}=$ Pick's disease, $\mathrm{PSP}=$ progressive supranuclear palsy, $\mathrm{CBD}=$ corticobasal degeneration. Atrophy graded as: relatively spared $(-)$, mildly involved $(+)$, moderately impaired $(++)$, severely impaired $(+++) . \mathrm{PFC}=$ prefrontal cortex; $\mathrm{PMC}=$ premotor cortex; $\mathrm{TL}=$ temporal lobe, med $=$ medial, lat $=$ lateral $; \mathrm{PL}=$ parietal lobe $; \mathrm{OL}=$ occipital lobe; $\mathrm{STR}=$ striatum; $\mathrm{CRB}=$ cerebellum. $\mathrm{LR}$ y $=$ atrophy associated with left/right asymmetry. 
The existence of typical atrophy patterns brought to the creation of automatic classificators, able to discriminate with good or excellent accuracy $(>80 \%)$ between two diagnostic categories (AD from controls, AD from FTLD and so on). The main shortcoming of these systems is their lack of applicability in a real clinical setting, as often they do not communicate to the medical operator the criteria used to determine the classification ${ }^{22}$.

The criteria should also not be too abstract, and the classificators should not be limited to a clear-cut binary choice, but be able to decide between multiple plausible diagnostic hypotheses. A recent attempt was made to create a real three classes classifier ${ }^{23}$ able to discriminate AD, FTLD and healthy controls.

A good index to discriminate ${ }^{24,25}$ bvFTD from AD is given by the MRI volumes of the caudate and gyrus rectus at the clinical onset of the diseases. Following the longitudinal evolution (1-2 years), many brain areas decrease in volume in dementia patients more than in healthy subjects, but only the volume of orbitofrontal cortex can discriminate between AD and FTLD, as the decrease is greater in FTLD. Even if White Matter (WM) volume reduction is greater in FTLD than in AD, this effect is not significant and cannot be used to distinguish them. While FTLD has a greater Grey Matter (GM) atrophy rate per year (3.6\% FTLD, 2.5\% AD, $1 \%$ healthy controls), just as with WM volume reduction this values is not robust enough to be used in diagnosis, and the volumes at the onset of the disease seem to be the more useful discriminant index.

A simple qualitative scale ${ }^{26}$ measuring atrophy $(0-4,0=$ no atrophy, $4=$ severe atrophy) showed a good potential in the differentiation between different pathological forms (accuracy 75-84\%) and between controls, ALS and FTLD patients (accuracy 81-95\%). The areas to be analyzed with this rating scale are the motor cortex, cingulate cortex, orbitofrontal cortex and anterior temporal cortex. Higher atrophy values characterize all FTLD forms, with more marked orbitofrontal atrophy (but less marked in motor areas) in bvFTD, and with the opposite pattern in FTD-ALS. In ALS, only the atrophy of the orbitofrontal cortex is evident in ALS, and to a lesser extent than in bvFTD. In general, atrophy is more widespread in bvFTD than in ALS when considering non motor areas ${ }^{27}$. 
SPECT and PET usefulness in differential diagnosis of dementia is ascertained ${ }^{28}$, and their ability to discriminate between different disorders can be greater than MRI, as shown by a study that examined 160 patients diagnosed with FTLD, AD or vascular dementia ${ }^{29}$. A frontal hypometabolism or hypoperfusion could help in distinguish between FTLD and AD. Recent reviews ${ }^{28,30}$, that compared $342 \mathrm{AD}$ and 112 FTLD patients calculated that PET has 98-99\% sensitivity and $65-85 \%$ specificity, while for SPECT the values are $72-80 \%$ (sensitivity) and 78 $81 \%$ (specificity), computed on $480 \mathrm{AD}$ and 158 FTLD patients. When extending the patients groups to include DLB, PET ratings drop to $71 \%$ for sensitivity and $65 \%$ for specificity ${ }^{28}$.

There are not many experiments comparing PET and SPECT on the same patients: even if PET seems to be a slightly better technique (better spatial resolution), the answer is not conclusive and SPECT has the advantage of being the less expensive of the two exams. It has been shown that the agreement rate between the exams at a 18 months follow-up could be as good as $90 \%{ }^{31}$. In order to maximize the diagnostic ability, it is advisable ${ }^{32}$ to acquire simultaneously PET/SPECT images with high resolution anatomical images (CT or MRI), and use semi quantitative or quantitative analysis to compare the patients scan with control groups matched for age and sex. In Italy and other countries, the current laws do not allow the use of ionizing radiation on healthy subjects, and therefore often a center cannot acquire in house normative control data. It is however possible to use data gathered in different foreigner centers, losing about $10 \%$ of accuracy. This loss can be partially recovered using a spatial normalization procedure that can compensate for the signal difference between different scanners ${ }^{33}$.

The quantitative analysis should be also carried out with groups of patients diagnosed with different disorders, as it is possible to find atypical forms of non-FTLD dementia that can imitate perfusion patterns typical of FTLD, as it is shown in a study ${ }^{34}$ that found in the cerebrospinal fluid (CSF) of 9 out of 43 patients diagnosed with FTLD a protein pattern suggestive of AD. Also in AD there was a frontal hypoperfusion pattern when compared to healthy subjects, but when compared only with FTLD patients the area of hypoperfusion was more posterior ${ }^{34}$. Even if in current practice the 
request for a SPECT/PET scan is not accompanied by clinical or cognitive data, including this information in the statistical analysis or in the criteria to choose the control group can improve the precision of quantitative analysis. In fact, a SPECT study ${ }^{35}$ carried on 23 FTLD patients (13 with episodic memory deficits) found that the metabolic alterations correlated with the clinical severity of the disease, and the temporal lobe was more severely damaged in the group with memory deficits.

Cerebral WM is very anisotropic (that is, it has properties that depend on the direction along which they are measured, as for example wood, which is easier to split along its grain than against it), because axon are gathered in compact bundles, which form tracts with precise spatial orientation. By indirectly measuring the water diffusion in cerebral tissues it is possible to obtain estimates of WM anisotropy and reconstruct images of the principal tracts. Different techniques allow to reconstruct WM bundles starting from diffusion weighted images, and FA (fractional anisotropy) and MD (mean diffusivity) are among the most common index, calculated using the DTI model (Diffusion Tensor Imaging). When the bundles are altered by microstructural damages, dysfunction of axonal organization or by fiber loss caused by Wallerian degeneration, their FA decreases and their MD increases. Dementia patients and healthy controls can be clearly discerned using DTI metrics; furthermore, different dementias have different characteristic patterns: in FTLD damaged areas are more anterior and in $\mathrm{AD}$ more posterior ${ }^{36}$. The anterior cingulate showed a decreased FA in bvFTD patients, which correlated with executive deficits, while the posterior cingulate showed a decreased FA in AD patients, correlating with visual working memory deficits ${ }^{37}$.

A study considering 74 FTLD and 19 AD, classified by CSF protein pattern, comparing the diagnostic potential of studying WM microstructure and GM volumes, found that adding WM data increases both the sensibility and specificity of the exam from $81 \%$ to $89 \%{ }^{38}$. Another study showed that the analysis of the only cingulum WM bundle can be at least as indicative that studying GM volumes ${ }^{39}$. 
A particularly interesting neuroimaging metric is the Loss in Connectivity (LoCo), a measure that integrates the information from GM atrophy pattern and microstructural WM alteration in a single informative index ${ }^{40}$. LoCo calculates the percentage of altered WM bundles starting from cortical regions involved in FTLD and/or AD (orbitofrontal and subcortical for FTLD, temporo-parietal for $\mathrm{AD})$.

Studying WM integrity provides an excellent characterization of FTLD subtypes, especially the different forms of PPA, as in this illness linguistic deficit correlate with alterations of specific WM $\operatorname{tracts}^{41-43}$.

WM damage patterns generally partially border on GM ones, even if in FTLD it is more widespread, with a bilateral frontal involvement in bvFTD, asymmetric fronto-parieto-temporal in nfPPA, fronto-temporal in svPPA, fronto-parietal in lvPPA ${ }^{44}$. In FTD-ALS there is a pattern of WM and GM damage that lies roughly halfway between bvFTD, in which the damage to frontotemporal cortex areas and WM bundles is greater, and ALS with a greater impairment of motor areas and bundles ${ }^{45}$.

In bvFTD patients the corpus callosum shows widespread damage, while in svPPA the damage is limited to a small portion of the genu ${ }^{46}$. Damage in both svPPA and lvPPA is lateralized to the left hemisphere, but can be told apart for the different magnitude of damage in the dorsal and ventral systems, as in svPPA ${ }^{47}$ the damage is particularly evident in the uncinate fasciculus (that joins frontal and temporal areas), while in lvPPA the damage is more localized to the posterior part of corona radiata $^{48}$. The damage of aslant tract (a bundle that joins Broca's area with cingulate cortex and supplementary premotor areas) is typical of nfPPA ${ }^{47,49}$.

While linguistic production deficits correlate with alterations of frontal WM bundles (e.g. aslant tract), integrity of perisilvian areas and of their connectivity is crucial for syntactic processes ${ }^{50}$. Semantic deficits and comprehension impairment are correlated with alterations of the uncinate fasciculus ${ }^{47-49}$. Also the superior longitudinal fasciculus (SLF that joins temporo-parietal and frontal areas) and inferior longitudinal fasciculus (ILF that joins occipital and temporal areas) show 
differences in PPA: FA is decreased in the SLF in nfPPA, in the ILF in svPPA, while in lvPPA it is more similar to normal controls ${ }^{51}$.

Even if the patterns described in this section are reliable when performing statistical analysis at group level, superposition of different patterns are widespread and partially overlapping in different subtypes of FTLD ${ }^{52-54}$. However, a study ${ }^{55}$ enrolling 9 svPPA, 9 nfPPA and 9 AD found that at least in some cases DTI metrics can be accurate enough to support a diagnosis at single patient level.

\section{Patients neuroimaging characterization}

Functional characterization of FTLD allowed examining neural correlates of the main deficits observed in clinical practice: impaired emotion judgment, socially inadequate behavior, apathy, impaired mnestic functions and a general deficit in social skills.

Three different theories about the neural correlates of emotional processing have been investigated from the point of view of FTLD research ${ }^{56}$ : the right hemispherical specialization theory, the limbic system theory and the multimodal theory, which hypothesizes a network of brain areas in which some hub are specific for the elaboration of a specific emotion (e.g.: orbitofrontal cortex for anger, amygdala for fear, etc...). Recognition of faces that express emotions is usually damaged in bvFTD and svPPA, but are much more spared in nfPPA ${ }^{56}$. These deficits could be explained by temporal atrophy and especially atrophy of the fusiform gyrus, which is specialized in the elaboration and recognition of human faces, but the results reach statistical significance even when controlling for the decrease in the perceptual skills of patients. This finding is compatible with both hemispheric and limbic theories, as these areas are damaged in bvFTD and svPPA but relatively spared in nfPPA, but the differences in recognizing single emotions only partially agree with the multimodal model. It must however be recalled that a distribute network damaged in an important node could have similar deficits, regardless of the specific task of the damaged location. 
A recent fMRI study that enrolled 20 FTLD $^{57}$, partially agreeing with limbic and multimodal theories, found hypoactivation of the limbic system, fusiform gyrus, occipital cortex, prefrontal ventral cortex and insula in patients when they were watching faces showing disgust.

In another study the authors identified areas that in healthy subjects are synchronized with different areas of the amygdala, an important area in emotion processing ${ }^{58}$. They found that ventral amygdala is functionally connected with a network specialized in perception (including the fusiform gyrus and orbitofrontal cortex), medial amygdala is functionally connected with a network that includes the nucleus accumbens and is specialized in elaborating reward and gratifying stimuli, and the dorsal amygdala is connected with a network specialized in elaborating painful and unexpected stimuli (the so called Saliency Network, SLN, that includes the medial cingulate cortex and anterior insulae). Measuring the volume of the three networks in 20 FTLD patients, the authors found that they correlate specifically with different deficits: the pleasure network correlated with socioemotional withdrawal and inappropriate behavior, the SLN correlated with an inappropriate level of trust and the perceptual network with difficulties in recognizing and attending to social clues.

The link between orbitofrontal and medial prefrontal cortex dysfunctions and inadequate behavioral inhibition is highlighted by two PET and two Voxel Based Morphometry (VBM) studies ${ }^{59}$. It must however be considered that using different neuropsychological tests or behavioral measures can change significantly the results: therefore, the need to develop more reliable and simple tests, useful in differential diagnosis and that correlate robustly with neurobiological data. The Hayling Sentence Completion Test (which asks to complete a set of sentences with nonsense conclusions, suppressing the automatic answer, as in "The captain wanted to stay with the sinking... banana") was found to be adequate ${ }^{59}$, as it correlates with orbitofrontal cortex atrophy and can distinguish FTLD and AD in clinical practice.

Other interesting studies found correlations between basal ganglia and frontotemporal cortex volumes and apathy ${ }^{60}$, mind reading ${ }^{61}$ and overstatement of personal skills ${ }^{62}$. The impairment in 
self-monitoring correlates with the volume of the aforementioned areas only when considering overstatement in the judgments of self and not understatement ${ }^{62}$. Atrophy of specific subsystems correlates with specific monitoring functions: self-monitoring of emotional control correlates with orbitofrontal atrophy ${ }^{62}$, and empathic worries with the atrophy of anterior and polar temporal areas ${ }^{63}$. The social context network model (SCNM) suggests that the integration of the frontal (which is responsible for context analysis and predicting the future) and temporal cortex (semantic storage of context), mediated by the insula (comparing the external environment with internal models) could be crucial in identifying others' states of mind and emotions ${ }^{64}$. A study recruiting 12 bvFTD and 10 nfPPA agrees with SCNM, as in nfPPA these skills were more severely damaged and atrophy of fronto-temporal-insular cortex was more evident ${ }^{65}$.

Mnestic abilities are impaired in FTLD patients, and even if the characteristics of the damage and atrophy patterns are usually different from $\mathrm{AD}$, in some cases there is significant overlap between clinical symptoms of the two pathologies. For instance, in working memory tasks the performance can be equally impaired in $\mathrm{AD}$ and FTLD, as this function has its neural correlates in a frontoparietal executive network which can be dysfunctional both when the damage is localized to a frontal node (as in FTLD) and when it is localized to a parietal one (AD), even if must be noted that the qualitative aspects of the deficits in AD and FTLD are quite different ${ }^{66}$. The differences have been the object of a study ${ }^{67}$ that enrolled 22 bvFTD, $32 \mathrm{AD}$ and 35 healthy controls and tried to correlate GM volumes with the scores obtained in specific neuropsychological tests. The scores in word list learning, immediate and delayed recall and recognition with or without interference correlated with temporal areas in both AD and bvFTD, while frontal functions as verbal fluency, verbal working memory and spatial working memory correlated with volumes of dorsolateral frontal cortex; other frontal functions as behavioral inhibition, risk assessment and recognition of emotions correlated with ventral medial frontal cortex. While memory and 'lateral' functions deficits are present both in $\mathrm{AD}$ and FTLD (with a non-significant trend indicating greater impairment in $\mathrm{AD}$ ), 'medial' deficits are typical of bvFTD ${ }^{67}$, as striatal dysfunction in associative 
learning ${ }^{68}$. Even if the impairment of memory is of a similar magnitude in the two groups, it has been associated to different patterns of atrophy and possibly to two different mechanisms ${ }^{67}$.

A recurring theme in the study of dementias is the analysis of a group of brain areas functionally integrated in fronto-striatal networks ${ }^{69}$. This line of study tied to characterize the functional correlates of social, cognitive and emotive deficits.

Recently ${ }^{70}$ it has been observed that some cerebral alterations can be identified also by studying the resting connectivity (or synchrony), as measured in Resting State imaging. In particular, the connectivity in the frontal executive networks is reduced, even when controlling for the atrophy found in FTLD. The SLN, important in the detection of highly salient and unexpected external stimuli (Figure 2) showed a reduction in connectivity, as previously stated by other studies ${ }^{71}$, while the medial prefrontal cortex was hyperconnected, especially in bvFTD patients. The authors proposed a frontolimbical disconnection accompanied by a maladaptive (it correlated with index such as disinhibition and apathy) frontal hyperconnection, possibly due to the lack of the input from the limbic system ${ }^{70}$. This finding was in agreement with a recent paper ${ }^{72}$ that tried to estimate the number of von Economo neurons in the anterior cingulate cortex (ACC), and found a more significant decrease in FTLD patients than in healthy controls and AD patients. The difference between $\mathrm{AD}$ and FTLD patients is only significant in the early stages of the pathology, and there is no difference when considering the pathological subtypes (it must be noted that the groups were very small). This could mean that VEN are attacked by a common final pathway in all proteinopathies and selectively hit in the early stages or, alternatively, that counting VEN is more difficult in the late stages and the higher vulnerability of frontal areas compared to the posterior ones can explain the relative difference in the loss of VEN, as these neurons are present in greater numbers in the cingulate cortex and anterior insulae, both nodes of the SLN system (Figure 1).

Graph theory, a mathematical technique used to study the structure of sets of connected objects (i.e., neural areas that compose a network) can help in the study of dementias by identifying the areas involved in a great number of connections (hub) and the type of the connection itself (long or short 
range). Using this approach it was shown ${ }^{73}$ that the structure of Resting State connectivity is damaged in bvFTD patients, in which only hubs located in the posterior areas were spared. Furthermore, disruption of the frontal network correlates with dysexecutive deficits.

The Default Mode Network (DMN, Figure 1) is composed by areas that are generally more activated during rest, symmetrically to the Executive Control Network (ECN, Figure 1), which is most active when we turn our attention externally in an active task. The DMN areas are also activated when we project ourselves, for example, imagining, simulating a future situation, trying to put ourselves in the perspective of others ${ }^{74}$. The $\mathrm{DMN}$ undergoes to opposite alterations in $\mathrm{AD}$ and bvFTD: the posterior nodes are progressively unconnected in AD and hyperconnected in bvFTD ${ }^{74}$.

SLN

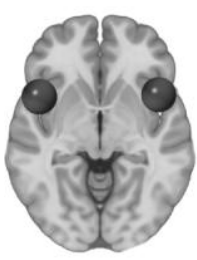

A

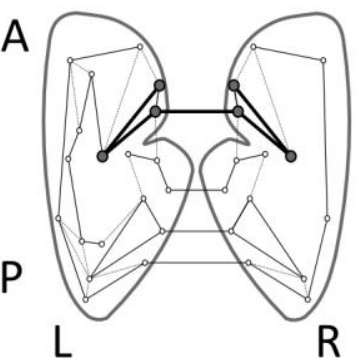

DMN
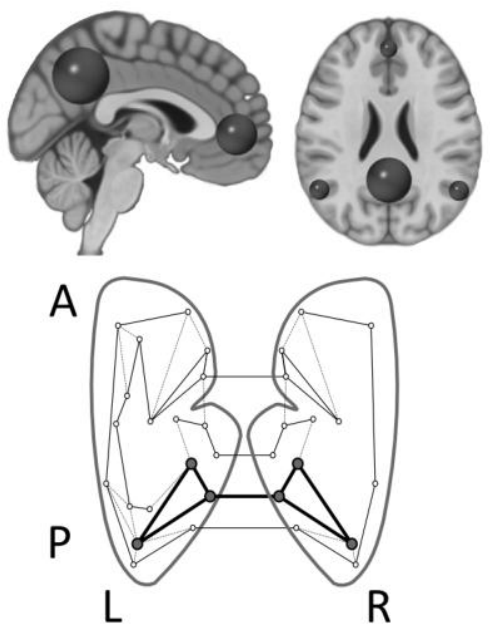

ECN
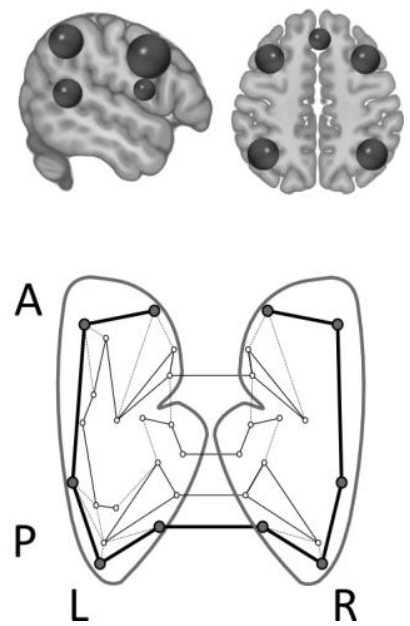

Figure 2. Intrinsic Networks of brain Resting State showed the main nodes in orthogonal projections (first row) and schematic flatted representation (second row). SLN = Salience Network, DMN = Default Mode Network. ECN = Executive Control Network. Adapted from ${ }^{75}$.

The deficits observed during the Resting State, sometimes quite specific, are particularly useful, as they can be measured even in non-cooperating patients and in patients with behavioural alterations, and might be seen as fingerprints of different forms of dementia ${ }^{75}$. Furthermore, it was noted ${ }^{76}$ that 
the main neurodegenerative patterns of FTLD and AD are uncannily similar to the networks identified with graph analysis in healthy control subjects.

It has been suggested ${ }^{77}$ that if neurodegeneration selectively damages the intrinsic connectivity network found in healthy subjects, then it is possible that the proteins responsible for the neural damages do actually "travel" along these networks during the evolution of the disease. It is therefore of the utmost importance to track the spreading of proteinopathies inside the networks (Figure 1) in order to understand and identify the most probable mechanism of transmission: prionslike, lack of tropism, cell signalling, axonal transport, selective vulnerability of some cells or connections.

This 'deep phenotyping' of the evolution of dementias from the network graph analysis perspective has been called the study of molecular nexopaties (from Latin nectere, tie). The study of the characteristic of the spreading of the disease is particularly important: from the initial damage of a node, the illness spreads and impairs other nodes using long connections or short ones? Does the spreading have a gradient (for instance between the two hemispheres)? Is there a global change of the whole network or a gradual transmission from the entry point? The answer to these questions could shed light on the nature of the mechanisms of diseases transmission. For example, a large database of healthy subjects Resting State were analyzed using the graph-analysis of the intrinsic connections searching for pattern of connectivity with the greatest capacity to recapitulate the pattern of neurodegeneration of $\mathrm{AD}$, bvFTD, nfPPA, svPPA, CBS ${ }^{78}$. The processes of disease spreading could be mediated by one (or more) of the following alternative mechanisms linked to different kind of connectivity profiles:

1) Lack of neurotrophism = vulnerability of the less connected nodes;

2) Neurotransmitter-mediated spreading = vulnerability of 'epicenter' nodes, characterized by short-distance links with all other nodes;

3) Specific vulnerability of the hubs, due to the elevated connection activity;

4) Vulnerability of neural subpopulations, not linked to specific topological correlates. 
The results of the study lend support to the second hypothesis ${ }^{78}$.

\section{New neuroimaging tools}

ASL is a relatively recent MRI technique that in the last years has evolved enough to be considered for use in clinical practice. Its working principle is simple: it inverts the signal of the water inside the arteries and labels it, thereby creating an "endogenous marker". Labeled blood perfuses the brain and the signal generated by brain tissues added to the signal of the blood flowing is acquired. A second scan acquires an image of the brain without labeling the blood, and the two images are subtracted to each other to obtain an estimate of blood perfusion ${ }^{79}$. In a consensus paper Alsop and collaborators suggested the optimal parameters (pseudo-continuous labelling, background suppression, 3D segmented reading and quantitative analysis) to use ASL in clinical practice, in order to keep homogeneity and comparability between studies ${ }^{79}$. Using ASL it is possible to obtain perfusion weighted images, like when using Nuclear Medicine techniques, without the use of ionizing radiations. Comparing ASL and perfusional SPECT on 68 patients with suspected AD, a paper ${ }^{80}$ found that the two techniques reach a similar accuracy, even if ASL is more prone to errors due to the greater influence of hemodynamic factors (accounting for $70 \%$ of misdiagnosis). Furthermore, in ASL the patterns of MRI hypoperfusion, cerebral atrophy and WM alterations reach a good level of agreement, as shown by a multimodal study using ASL, DTI and VBM on 20 AD, 20 FTLD and 21 control subjects ${ }^{81}$. Other studies showed that ASL can distinguish reliably between FTLD, DLB and $\mathrm{AD}^{82}$ and that its results can be used as useful functional correlates in $\operatorname{research}^{83}$.

The set of possible new radiopharmaceuticals is quite large, and it is likely that the next years will see the discovery of new tracers with useful applications in the diagnosis of dementia. The development of new in vivo measures, directly linked to neuropathological correlates is of particular interest. To this day, two radiopharmaceuticals that can label $\beta$-amyloid plaques have been developed: Pittsburgh compound B (11C-PiB) and Florbetapir(18F), or 18F-AV-45. While 
plaques are typically associated with $\mathrm{AD}$, combining $\beta$-amyloid imaging with PET-FDG can increase the potential of differential diagnosis. In 154 patients, mostly affected by AD, FTLD and DLB, labeling amyloid plaques was found to be useful in reconsidering some uncertain diagnosis. FDG alone is usually enough to reach a diagnosis, but as atypical cases are more likely to be misdiagnosed, an exam using $\mathrm{PiB}$ as tracer can be useful. Furthermore, being positive for both $\mathrm{PiB}$ and FDG can predict the progression of patients to dementia, and the diagnosis formulated on the grounds of the results of this exams are not changed in $96 \%$ of cases ${ }^{84}$ at 2 years follow-up.

Between other radiopharmaceuticals in development, the ones that can be used to perform neuroimaging of inflammation are of particular interest. These molecules map the translocator protein 18 kDa (TSPO): 18F-PBR111, 11C- 18F- PBR06, 11C- 18F- PBR28 and 18F-FEPPA ${ }^{85}$, and allow the clinician to have a map of microglia activation, linked with the inflammatory response both in time and space.

Other molecules [18F]-T807, [18F]-T808, [18F]-THK5105 and [18F]-THK523 ${ }^{85}$ can label the accumulations of tau protein and are therefore of particular interest as are other radiopharmaceuticals currently in development, that should be able to mark selectively the enzymes involved in the biological pathways that create the anomalous protein accumulations ${ }^{85}$.

Another research branch is focused on the development of new radiopharmaceuticals able to label the receptors of neurotransmitters, selectively involved in different dementia types. An example of this approach is the use of molecules like 123I-FP-CIT, 123I- $\beta$-CIT, previously only used in Parkinson-like diseases. This could be useful as dopaminergic neurons of basal ganglia are compromised in different ways in AD, FTLD and DLB: in the majority of DLB cases there are alterations, as in about $50 \%$ of FTLD cases, while in AD they are almost always absent. FP-CIT can therefore be used to exclude DLB, even if this evidence is not always strong enough to discriminate between FTLD and $\mathrm{AD}^{86}$. False positives and negatives can be due to drug intake or vascular problems in suspected dementias ${ }^{87}$. 


\section{Presymptomatic patients}

One of the most interesting aspects in the study of patients carrying a mutation linked to FTLD is the possibility of studying in vivo the evolution of subjects before the pathology becomes clinically relevant and thus to identify which biomarkers can better predict the onset of the disease and monitor the response to experimental pharmacological treatments ${ }^{88}$. Studies on presymptomatic patients are still in a very early stage, but some authors found what could be an important result: similarly to presymptomatic familiar AD patients, also in FTLD functional alterations in perfusion ${ }^{89}$ or Resting State ${ }^{90}$, similar to the ones found in symptomatic patients, could be present in asymptomatic patients many years before the onset of the illness. However, it must be noted that no one still found truly significant alterations in GM or WM, and that the sample sizes were too small to draw conclusions at the current stage.

It should also be noted that a study ${ }^{91}$ found in asymptomatic subjects carriers of the MAPT mutation alterations in the values of MI and NAA. The values found in asymptomatic subjects, years before the disease onset, were roughly halfway between the ones of healthy subjects and of patients with diagnosed dementia.

During the last years new imaging techniques in the diagnosis of dementias have been investigated by a number of papers, thus opening new diagnostic approaches to these illnesses. In particular, the techniques more often investigated are the study of microstructural and axonal integrity of WM (DWI) and functional correlates (fMRI, Resting State) of functions damaged by FTLD. Furthermore, recent advances in a MRI technique able to obtain perfusional weighted images, called Arterial Spin Labelling (ASL), brought it to the point of being comparable with perfusional SPECT. ASL advantage over the latter is the absence of ionizing radiations and of their noxious effects; therefore, it is a significant improvement for patients, especially when they are being monitored for years to follow the evolution of a degenerative disease. 


\section{Conclusions}

While the current approach to the study of dementias is mostly focused on mapping and correlating atrophy of cerebral areas and cognitive functions, the results are often as elaborate as they are unreliable because of the variability in forms and manifestations of dementia and the relatively high variance in the atrophy patterns. A better approach would be to focus on the possible treatments for the different syndromes, and link with a rational and well-thought master plan the different aspects: genetics, clinical observations and etiopathology.

Neuroimaging techniques have shown to be useful supporting tools in diagnosis, even if not always determinant to reach a conclusive decision, and quite important to identify phenocopies. Future neuroimaging studies should strive to include at least CSF data, and possibly also genetic and pathological information, in order to avoid grouping patients with different (misdiagnosed) pathologies.

At the moment there is not a neuroimaging biomarker that could track the progressive course of dementias and the effect of therapies, but it is possible that in the future DTI and molecular imaging could fill this void. Monitoring in vivo the evolution of the pathology is essential, but to the best of our knowledge no study embarked in this huge effort (ideally, ASL, volumetry, Resting State and DTI every 3 months for at least 5 years on a large and well-defined group of patients, plus neuropsychological testing, clinical observation and CSF analysis), which would only be possible in a large multicentric study, while asymptomatic forms would require even longer observation periods. 


\section{References}

1. Rohrer JD, Rosen HJ. Neuroimaging in frontotemporal dementia. Int Rev Psychiatry. Informa Healthcare USA; 2013;25(2):221-9.

2. Seltman RE, Matthews BR. Frontotemporal lobar degeneration: epidemiology, pathology, diagnosis and management. CNS Drugs. 2012;26(10):841-70.

3. Hu WT, Seelaar H, Josephs KA, Knopman DS, Boeve BF, Sorenson EJ, et al. Survival Profiles of Patients With Frontotemporal Dementia and Motor Neuron Disease. Arch Neurol. 2009;66(11):1359-64.

4. Hodges JR, Davies R, Xuereb J, Kril J, Halliday G. Survival in frontotemporal dementia. Neurology. 2003 Aug;61(3):349-54.

5. Kirshner HS. Primary progressive aphasia and Alzheimer's disease: brief history, recent evidence. Curr Neurol Neurosci Rep. 2012;12(6):709-14.

6. Kirshner HS. Frontotemporal dementia and primary progressive aphasia, a review. Neuropsychiatr Dis Treat. 2014;10:1045-55.

7. Kurz A, Kurz C, Ellis K, Lautenschlager NT. What is frontotemporal dementia? Maturitas. 2014;79(2):216-9.

8. Halliday G, Bigio EH, Cairns NJ, Neumann M, Mackenzie IRA, Mann DMA. Mechanisms of disease in frontotemporal lobar degeneration: gain of function versus loss of function effects. Acta Neuropathol. 2012;124(3):373-82. 
9. Riedl L, Mackenzie IR, Förstl H, Kurz A, Diehl-Schmid J. Frontotemporal lobar degeneration: current perspectives. Neuropsychiatr Dis Treat. 2014;10:297-310.

10. Rascovsky K, Hodges JR, Knopman D, Mendez MF, Kramer JH, Neuhaus J, et al. Sensitivity of revised diagnostic criteria for the behavioural variant of frontotemporal dementia. Brain. 2011;134(Pt 9):2456-77.

11. Gorno-Tempini ML, Hillis AE, Weintraub S, Kertesz A, Mendez M, Cappa SF, et al. Classification of primary progressive aphasia and its variants. Neurology. 2011;76(11):100614.

12. Chare L, Hodges JR, Leyton CE, McGinley C, Tan RH, Kril JJ, et al. New criteria for frontotemporal dementia syndromes: clinical and pathological diagnostic implications. $\mathbf{J}$ Neurol Neurosurg Psychiatry. 2014;85(8):865-70.

13. Park HK, Chung SJ. New perspective on parkinsonism in frontotemporal lobar degeneration. J Mov Disord. Korean Movement Disorders Society. 2013;6(1):1-8.

14. Kipps CM, Hodges JR, Hornberger M. Nonprogressive behavioural frontotemporal dementia: recent developments and clinical implications of the "bvFTD phenocopy syndrome”. Curr Opin Neurol. 2010;23(6):628-32.

15. Rohrer JD. Structural brain imaging in frontotemporal dementia. Biochim Biophys Acta. 2012;1822(3):325-32.

16. Whitwell JL, Josephs KA. Recent advances in the imaging of frontotemporal dementia. Curr Neurol Neurosci Rep. 2012;12(6):715-23. 
17. Whitwell JL, Przybelski SA, Weigand SD, Ivnik RJ, Vemuri P, Gunter JL, et al. Distinct anatomical subtypes of the behavioural variant of frontotemporal dementia: a cluster analysis study. Brain. 2009;132(Pt 11):2932-46.

18. Hartikainen P, Räsänen J, Julkunen V, Niskanen E, Hallikainen M, Kivipelto M, et al. Cortical thickness in frontotemporal dementia, mild cognitive impairment, and Alzheimer's disease. J Alzheimers Dis. 2012;30(4):857-74.

19. Basely M, Ceccaldi M, Boyer L, Mundler O, Guedj E. Distinct patterns of medial temporal impairment in degenerative dementia: a brain SPECT perfusion study in Alzheimer's disease and frontotemporal dementia. Eur J Nucl Med Mol Imaging. 2013;40(6):932-42.

20. Souza L De, Chupin M, Bertoux M. Is hippocampal volume a good marker to differentiate Alzheimer's disease from frontotemporal dementia? Alzheimer's Dis. 2013;36(1):57-66.

21. Muñoz-Ruiz MÁ, Hartikainen P, Koikkalainen J, Wolz R, Julkunen V, Niskanen E, et al. Structural MRI in frontotemporal dementia: comparisons between hippocampal volumetry, tensor-based morphometry and voxel-based morphometry. PLoS One. 2012;7(12):e52531.

22. Abdi H, Williams L, Beaton D. Analysis of Regional Cerebral Blood Flow Data to Discriminate among Alzheimer's Disease, Frontotemporal Dementia, and Elderly Controls: A Multi-Block Barycentric. J Alzheimer’s. 201231 Suppl 3:S189-201.

23. Raamana PR, Rosen H, Miller B, Weiner MW, Wang L, Beg MF. Three-Class Differential Diagnosis among Alzheimer Disease, Frontotemporal Dementia, and Controls. Front Neurol. 2014;5:71.

24. Lam BYK, Halliday GM, Irish M, Hodges JR, Piguet O. Longitudinal white matter changes in frontotemporal dementia subtypes. Hum Brain Mapp. 2014;35(7):3547-57. 
25. Frings L, Yew B, Flanagan E, Lam BYK, Hüll M, Huppertz H-J, et al. Longitudinal grey and white matter changes in frontotemporal dementia and Alzheimer's disease. PLoS One. 2014;9(3):e90814.

26. Ambikairajah A, Devenney E, Flanagan E, Yew B, Mioshi E, Kiernan M, et al. A visual MRI atrophy rating scale for the amyotrophic lateral sclerosis-frontotemporal dementia continuum. Amyotroph Lateral Scler Front Degener. 2014;3-4:226-34.

27. Rajagopalan V, Pioro EP. Distinct patterns of cortical atrophy in ALS patients with or without dementia: an MRI VBM study. Amyotroph Lateral Scler Frontotemporal Degener. 2014;15(3-4):216-25.

28. Davison C, O'Brien J. A comparison of FDG-PET and blood flow SPECT in the diagnosis of neurodegenerative dementias: a systematic review. Int J Geriatr Psychiatry. 2014;29(6):55161.

29. Hoffmann M. Frontal network syndrome testing: clinical tests and positron emission tomography brain imaging help distinguish the 3 most common dementia subtypes. Am $\mathbf{J}$ Alzheimers Dis Other Demen. 2013;28(5):477-84.

30. Yeo JM, Lim X, Khan Z, Pal S. Systematic review of the diagnostic utility of SPECT imaging in dementia. Eur Arch Psychiatry Clin Neurosci. 2013;263(7):539-52.

31. Tripathi M, Tripathi M, Damle N, Kushwaha S, Jaimini A, D’Souza MM, et al. Differential diagnosis of neurodegenerative dementias using metabolic phenotypes on F-18 FDG PET/CT. Neuroradiol J. 2014;27(1):13-21.

32. Jolepalem P, Wu D. Semantic Dementia Diagnosed by F-18 FDG PET/MRI: Co-registered Images. J Clin Imaging Sci. 2013;3:35. 
33. Thiele F, Young S, Buchert R, Wenzel F. Voxel-based classification of FDG PET in dementia using inter-scanner normalization. Neuroimage. 2013;77:62-9.

34. Padovani A, Premi E, Pilotto A, Gazzina S, Cosseddu M, Archetti S, et al. Overlap between frontotemporal dementia and Alzheimer's disease: cerebrospinal fluid pattern and neuroimaging study. J Alzheimers Dis. 2013;36(1):49-55.

35. M. Papma J. Episodic Memory Impairment in Frontotemporal Dementia; A 99mTcHMPAO SPECT Study. Curr Alzheimer Res. 2014;10(3):332-9.

36. Lu PH, Lee GJ, Shapira J, Jimenez E, Mather MJ, Thompson PM, et al. Regional differences in white matter breakdown between frontotemporal dementia and early-onset Alzheimer's disease. J Alzheimers Dis. 2014;39(2):261-9.

37. Tartaglia MC, Zhang Y, Racine C, Laluz V, Neuhaus J, Chao L, et al. Executive dysfunction in frontotemporal dementia is related to abnormalities in frontal white matter tracts. J Neurol. 2012;259(6):1071-80.

38. McMillan CT, Avants BB, Cook P, Ungar L, Trojanowski JQ, Grossman M. The power of neuroimaging biomarkers for screening frontotemporal dementia. Hum Brain Mapp. 2014;35(9):4827-40.

39. Santillo AF, Mårtensson J, Lindberg O, Nilsson M, Manzouri A, Landqvist Waldö M, et al. Diffusion tensor tractography versus volumetric imaging in the diagnosis of behavioral variant frontotemporal dementia. PLoS One. 2013;8(7):e66932.

40. Kuceyeski A, Zhang Y, Raj A. Linking white matter integrity loss to associated cortical regions using structural connectivity information in Alzheimer's disease and fronto-temporal dementia: the Loss in Connectivity (LoCo) score. Neuroimage. 2012;61(4):1311-23. 
41. Ash S, Evans E, O’Shea J, Powers J, Boller A, Weinberg D, et al. Differentiating primary progressive aphasias in a brief sample of connected speech. Neurology. 2013;81(4):329-36.

42. Grossman M, Powers J, Ash S, McMillan C, Burkholder L, Irwin D, et al. Disruption of large-scale neural networks in non-fluent/agrammatic variant primary progressive aphasia associated with frontotemporal degeneration pathology. Brain Lang. 2013;127(2):106-20.

43. Charles D, Olm C, Powers J, Ash S, Irwin DJ, McMillan CT, et al. Grammatical comprehension deficits in non-fluent/agrammatic primary progressive aphasia. $\mathrm{J}$ Neurol Neurosurg Psychiatry. 2014;85(3):249-56.

44. Agosta F, Scola E, Canu E, Marcone A, Magnani G, Sarro L, et al. White matter damage in frontotemporal lobar degeneration spectrum. Cereb Cortex. 2012;22(12):2705-14.

45. Lillo P, Mioshi E, Burrell JR, Kiernan MC, Hodges JR, Hornberger M. Grey and white matter changes across the amyotrophic lateral sclerosis-frontotemporal dementia continuum. PLoS One. 2012;7(8):e43993.

46. Walterfang M, Luders E, Looi JCL, Rajagopalan P, Velakoulis D, Thompson PM, et al. Shape analysis of the corpus callosum in Alzheimer's disease and frontotemporal lobar degeneration subtypes. J Alzheimers Dis. 2014;40(4):897-906.

47. Mandelli ML, Caverzasi E, Binney RJ, Henry ML, Lobach I, Block N, et al. Frontal white matter tracts sustaining speech production in primary progressive aphasia. $\mathrm{J}$ Neurosci. 2014;34(29):9754-67.

48. Powers JP, McMillan CT, Brun CC, Yushkevich PA, Zhang H, Gee JC, et al. White matter disease correlates with lexical retrieval deficits in primary progressive aphasia. Front Neurol. 2013;4:212. 
49. Catani M, Mesulam MM, Jakobsen E, Malik F, Martersteck A, Wieneke C, et al. A novel frontal pathway underlies verbal fluency in primary progressive aphasia. Brain. 2013;136(Pt 8):2619-28.

50. Wilson SM, Galantucci S, Tartaglia MC, Gorno-Tempini ML. The neural basis of syntactic deficits in primary progressive aphasia. Brain Lang. 2012;122(3):190-8.

51. Galantucci S, Tartaglia MC, Wilson SM, Henry ML, Filippi M, Agosta F, et al. White matter damage in primary progressive aphasias: a diffusion tensor tractography study. Brain. 2011;134(Pt 10):3011-29.

52. Schwindt GC, Graham NL, Rochon E, Tang-Wai DF, Lobaugh NJ, Chow TW, et al. Wholebrain white matter disruption in semantic and nonfluent variants of primary progressive aphasia. Hum Brain Mapp. 2013;34(4):973-84.

53. Agosta F, Galantucci S, Canu E, Cappa SF, Magnani G, Franceschi M, et al. Disruption of structural connectivity along the dorsal and ventral language pathways in patients with nonfluent and semantic variant primary progressive aphasia: a DT MRI study and a literature review. Brain Lang. 2013;127(2):157-66.

54. Tovar-Moll F, de Oliveira-Souza R, Bramati IE, Zahn R, Cavanagh A, Tierney M, et al. White matter tract damage in the behavioral variant of frontotemporal and corticobasal dementia syndromes. PLoS One. 2014;9(7):e102656.

55. Sajjadi SA, Acosta-Cabronero J, Patterson K, Diaz-de-Grenu LZ, Williams GB, Nestor PJ. Diffusion tensor magnetic resonance imaging for single subject diagnosis in neurodegenerative diseases. Brain. 2013;136(Pt 7):2253-61. 
56. Kumfor F, Piguet O. Disturbance of emotion processing in frontotemporal dementia: a synthesis of cognitive and neuroimaging findings. Neuropsychol Rev. 2012;22(3):280-97.

57. Virani K, Jesso S, Kertesz A, Mitchell D, Finger E. Functional neural correlates of emotional expression processing deficits in behavioural variant frontotemporal dementia. J Psychiatry Neurosci. 2013;38(3):174-82.

58. Bickart KC, Brickhouse M, Negreira A, Sapolsky D, Barrett LF, Dickerson BC. Atrophy in distinct corticolimbic networks in frontotemporal dementia relates to social impairments measured using the Social Impairment Rating Scale. J Neurol Neurosurg Psychiatry. 2014;85(4):438-48.

59. O'Callaghan C, Hodges JR, Hornberger M. Inhibitory dysfunction in frontotemporal dementia: a review. Alzheimer Dis Assoc Disord. 2013;27(2):102-8.

60. Eslinger PJ, Moore P, Antani S, Anderson C, Grossman M. Apathy in frontotemporal dementia: behavioral and neuroimaging correlates. Behav Neurol. 2012;25(2):127-36.

61. Hodges J. Alzheimer's disease and the frontotemporal dementias: contributions to clinicopathological studies, diagnosis, and cognitive neuroscience. J Alzheimer's Dis. 2013;33 Suppl 1:S211-7.

62. Shany-Ur T, Lin N, Rosen HJ, Sollberger M, Miller BL, Rankin KP. Self-awareness in neurodegenerative disease relies on neural structures mediating reward-driven attention. Brain. 2014;137(Pt 8):2368-81.

63. Sollberger M, Rosen HJ, Shany-Ur T, Ullah J, Stanley CM, Laluz V, et al. Neural substrates of socioemotional self-awareness in neurodegenerative disease. Brain Behav. 2014;4(2):20114. 
64. Ibañez A, Manes F. Contextual social cognition and the behavioral variant of frontotemporal dementia. Neurology. 2012;78(17):1354-62.

65. Couto B, Manes F, Montañés P, Matallana D, Reyes P, Velasquez M, et al. Structural neuroimaging of social cognition in progressive non-fluent aphasia and behavioral variant of frontotemporal dementia. Front Hum Neurosci. Frontiers; 2013;7:467.

66. Stopford CL, Thompson JC, Neary D, Richardson AMT, Snowden JS. Working memory, attention, and executive function in Alzheimer's disease and frontotemporal dementia. Cortex. 2012;48(4):429-46.

67. Wong S, Flanagan E, Savage G, Hodges JR, Hornberger M. Contrasting prefrontal cortex contributions to episodic memory dysfunction in behavioural variant frontotemporal dementia and Alzheimer's disease. PLoS One. 2014;9(2):e87778.

68. Dalton MA, Weickert TW, Hodges JR, Piguet O, Hornberger M. Impaired acquisition rates of probabilistic associative learning in frontotemporal dementia is associated with frontostriatal atrophy. NeuroImage Clin. 2012;2:56-62.

69. Looi JCL, Walterfang M, Velakoulis D, Macfarlane MD, Svensson LA, Wahlund L-O. Frontotemporal dementia as a frontostriatal disorder: neostriatal morphology as a biomarker and structural basis for an endophenotype. Aust N Z J Psychiatry. 2012;46(5):422-34.

70. Farb NAS, Grady CL, Strother S, Tang-Wai DF, Masellis M, Black S, et al. Abnormal network connectivity in frontotemporal dementia: evidence for prefrontal isolation. Cortex. 2013;49(7):1856-73. 
71. Zhou J, Greicius MD, Gennatas ED, Growdon ME, Jang JY, Rabinovici GD, et al. Divergent network connectivity changes in behavioural variant frontotemporal dementia and Alzheimer's disease. Brain. 2010;133(Pt 5):1352-67.

72. Santillo AF, Nilsson C, Englund E. von Economo neurones are selectively targeted in frontotemporal dementia. Neuropathol Appl Neurobiol. 2013;39(5):572-9.

73. Agosta F, Sala S, Valsasina P, Meani A, Canu E, Magnani G, et al. Brain network connectivity assessed using graph theory in frontotemporal dementia. Neurology. 2013;81(2):134-43.

74. Irish M, Piguet O, Hodges JR. Self-projection and the default network in frontotemporal dementia. Nat Rev Neurol. 2011;8(3):152-61.

75. Warren JD, Rohrer JD, Schott JM, Fox NC, Hardy J, Rossor MN. Molecular nexopathies: a new paradigm of neurodegenerative disease. Trends Neurosci. 2013;36(10):561-9.

76. Raj A, Kuceyeski A, Weiner M. A network diffusion model of disease progression in dementia. Neuron. 2012;73(6):1204-15.

77. Warren JD, Rohrer JD, Hardy J. Disintegrating brain networks: from syndromes to molecular nexopathies. Neuron. 2012;73(6):1060-2.

78. Zhou J, Gennatas ED, Kramer JH, Miller BL, Seeley WW. Predicting regional neurodegeneration from the healthy brain functional connectome. Neuron. 2012;73(6):121627.

79. Alsop DC, Detre JA, Golay X, Günther M, Hendrikse J, Hernandez-Garcia L, et al. Recommended implementation of arterial spin-labeled perfusion MRI for clinical 
applications: A consensus of the ISMRM perfusion study group and the European consortium for ASL in dementia. Magn Reson Med. 2014.

80. Takahashi H, Ishii K, Hosokawa C, Hyodo T, Kashiwagi N, Matsuki M, et al. Clinical application of 3D arterial spin-labeled brain perfusion imaging for Alzheimer disease: comparison with brain perfusion SPECT. AJNR Am J Neuroradiol. 2014;35(5):906-11.

81. Zhang Y, Schuff N, Ching C, Tosun D, Zhan W, Nezamzadeh M, et al. Joint assessment of structural, perfusion, and diffusion MRI in Alzheimer's disease and frontotemporal dementia. Int J Alzheimers Dis. 2011;2011:546871.

82. Binnewijzend MAA, Kuijer JPA, van der Flier WM, Benedictus MR, Möller CM, Pijnenburg YAL, et al. Distinct perfusion patterns in Alzheimer's disease, frontotemporal dementia and dementia with Lewy bodies. Eur Radiol. 2014;24(9):2326-33.

83. Grieder M, Crinelli RM, Jann K, Federspiel A, Wirth M, Koenig T, et al. Correlation between topographic N400 anomalies and reduced cerebral blood flow in the anterior temporal lobes of patients with dementia. J Alzheimers Dis. 2013;36(4):711-31.

84. Ossenkoppele R, Prins ND, Pijnenburg YAL, Lemstra AW, van der Flier WM, Adriaanse SF, et al. Impact of molecular imaging on the diagnostic process in a memory clinic. Alzheimers Dement. 2013;9(4):414-21.

85. Holland JP, Liang SH, Rotstein BH, Collier TL, Stephenson NA, Greguric I, et al. Alternative approaches for PET radiotracer development in Alzheimer's disease: imaging beyond plaque. J Labelled Comp Radiopharm. 2014;57(4):323-31.

86. Spehl TS, Frings L, Hellwig S, Weiller C, Hüll M, Meyer PT, et al. Role of Semiquantitative Assessment of Regional Binding Potential in 123I-FP-CIT SPECT for the Differentiation of 
Frontotemporal Dementia, Dementia With Lewy Bodies, and Alzheimer's Dementia. Clin Nucl Med. 2015.

87. Aarsland D, Ballard CG. Biomarkers for dementia diagnosis: differentiating DLB and FTD may be difficult. J Neurol Neurosurg Psychiatry. 2012;83(11):1037.

88. Rohrer JD, Warren JD, Fox NC, Rossor MN. Presymptomatic studies in genetic frontotemporal dementia. Rev Neurol (Paris). 2013;169(10):820-4.

89. Jacova C, Hsiung G-YR, Tawankanjanachot I, Dinelle K, McCormick S, Gonzalez M, et al. Anterior brain glucose hypometabolism predates dementia in progranulin mutation carriers. Neurology. 2013;81(15):1322-31.

90. Dopper EGP, Rombouts SARB, Jiskoot LC, den Heijer T, de Graaf JRA, de Koning I, et al. Structural and functional brain connectivity in presymptomatic familial frontotemporal dementia. Neurology. 2014;83(2):e19-26.

91. Kantarci K. Magnetic resonance spectroscopy in common dementias. Neuroimaging Clin N Am. 2013;23(3):393-406. 